\title{
Caracterização morfométrica da Bacia Hidrográfica do Riacho das Garças, Pernambuco, Brasil
} Morphometric characterization of Garças River watershed in Pernambuco, Brazil

\author{
ALVES, Adriana Thays Araújo. Mestrado/Engenharia Civil \\ Universidade Estadual do Maranhão - Campus Bacabal. R. Teixeira de Freitas, 2492 - 2706, Bacabal - MA - Brasil. \\ CEP: 65700-000. Telefone: (86) 98859.9020 / E-mail: adrianathaaraujo@gmail.com

\section{BARROS, Vitor Hugo de Oliveira. Mestrado/Engenharia Civil} \\ Instituto Federal do Sertão Pernambucano - Campus Serra Talhada. Rodovia PE 320, s/n - km 126, Zona Rural, \\ Serra Talhada - PE - Brasil. Caixa Postal 78 / Telefone: (81) 99280.5562 / E-mail: vitor.barros@ifsertao- \\ pe.edu.br
}

\section{RESUMO}

A caracterização morfométrica de bacias hidrográficas é de grande importância no entendimento do comportamento hidrológico destas e as ferramentas de geoprocessamento possibilitam uma elevada precisão e agilidade nessas análises. Nesse sentido, o presente estudo consistiu na análise morfométrica da Bacia Hidrográfica do Riacho das Garças (BHRG), Pernambuco, Brasil. Foram utilizados dados SRTM processadas com o auxílio do QGIS, sendo o TauDEM utilizado como ferramenta de extração de dados. Como resultados mais relevantes destacam-se o valor do Coeficiente de compacidade $\left(\mathrm{K}_{\mathrm{c}}\right)$ de 1,803, Fator de forma $\left(\mathrm{K}_{\mathrm{f}}\right)$ de 0,244 e o Índice de Circularidade $\left(I_{c}\right)$ de 0,303 , que indicam um formato alongado que favorece o escoamento superficial da BHRG. Pode-se ainda destacar que a BHRG se apresentou como de $5^{\circ}$ ordem, os valores da Densidade de Drenagem $\left(D_{d}\right)$ e hidrográfica $\left(D_{h}\right)$ foram baixos, 0,365 e 0,135 , respectivamente, e em relação ao relevo, a região possui baixas declividades e altitudes. Como conclusões, destaca-se a baixa propensão a ocorrência de enchentes em condições normais de precipitação devido ao formato alongado da bacia. Por outro lado, o relevo com baixas declividades, não favorece o escoamento superficial. Por fim, os parâmetros relativos à drenagem indicam que a bacia hidrográfica em estudo é pobremente drenada.

Palavras-chave: Geoprocessamento, Semiárido Brasileiro, Sensoriamento Remoto

\begin{abstract}
Morphometric analysis of watershed is of great importance for the understanding of their hydrological behavior and the geoprocessing tools allow high precision and agility in these analyzes. In this sense, this study consisted of a morphometric analysis of the Garças River Watershed (GRW), Pernambuco, Brazil. SRTM data was processed in the QGIS and the TauDEM was used as a data extraction tool. The most relevant results are the $\mathrm{Kc}$ value of 1.803 , Kf of 0.244 , and Ic of 0.303 , which indicates an elongated that favors GRW surface runoff. It should also be noted that the fluvial hierarchy GRW is of 5th order, the values of Dd and Dh were low, 0.365, and 0.135, respectively, and, in relation to the relief, the region has low slopes and low altitudes. In conclusion, it is important to highlight the low propensity to flooding under normal rainfall conditions due to the elongated of the basin. In contrast, the relief with low slopes does not favor the runoff. Finally, the drainage parameters indicate that the hydrographic basin has poor drainage.
\end{abstract}

keywords: Geoprocessing, Brazilian Semiarid, Remote Sensing 
ALVES, ATA; BARROS, VHO (2021)

caracterização morfométrica da Bacia Hidrográfica do Riacho das Garças, Pernambuco, Brasil

\section{Introdução}

As bacias hidrográficas consistem em uma captação natural de água. De modo mais específico, são áreas de terra em que as águas superficiais convergem para um único ponto em uma elevação mais baixa, o exutório da bacia (Rahaman et al., 2015). Ainda, pode-se dizer que estas são unidades geográficas importantes de gestão (Soni, 2016), podendo ser empregadas como unidade ideal no gerenciamento dos recursos naturais (Abdeta et al., 2020; Rahaman et al., 2015).

Nesse aspecto, Fideles et al. (2016) destacam que a bacia hidrográfica tem sido empregada como unidade de gestão da paisagem, em relação ao planejamento ambiental, principalmente no que se refere à gestão dos recursos hídricos, proporcionando melhor entendimento sobre fragilidade do solo em termos de uso e ocupação. Nesse sentido, muitos estudos são conduzidos justamente ao nível de bacia hidrográfica, seja tendência de precipitação nestas (Carvalho et al., 2019), extrapolação de dados de vazão (Lira e Cardoso, 2018) ou análise morfométrica (Sousa e Paula, 2016). No contexto do semiárido brasileiro, Sousa e Paula (2016) inferem sobre a relevância de estudos em bacias hidrográficas frente a escassez natural dos recursos hídricos da região.Quanto a análise morfométrica, diversos parâmetros para caracterização morfométrica de bacias hidrográficas foram consolidados ainda no século passado, em que se destacam, por exemplo, as contribuições de Horton $(1932,1945)$ e Strahler (1952, 1957, 1964). Mahala (2019) destaca que por meio dos parâmetros morfométricos relativos à área e ao relevo, o comportamento hidrológico e morfológico de qualquer bacia pode ser melhor compreendido. Esta análise é realizada para avaliação quantitativa de bacias hidrográficas e para planejamento e gestão de recursos hídricos (Soni, 2016).Atualmente o desenvolvimento do Sistema de Informação Geográfica (SIG) e técnicas de geoprocessamento proporcionam uma maior facilidade e precisão na análise morfométrica de bacias hidrográficas. Para Lopes et al. (2018), a introdução de informações de relevo em ambiente SIG permite o conhecimento de características em qualquer bacia hidrográfica. Essas informações do terreno podem ser obtidas, dentre outros, pelo Modelo Digital de Elevação (MDE). No MDE, as informações do relevo são representadas por meio de uma estrutura numérica de dados que corresponde à distribuição espacial da altitude e da superfície do terreno (Oliveira et al., 2010).

Nesse sentido, o objetivo principal desse estudo foi realizar a análise morfométrica da Bacia Hidrográfica do Riacho das Garças, em Pernambuco, no semiárido brasileiro, de forma a analisar o comportamento hidrológico da mesma, através de sensoriamento remoto e técnicas de geoprocessamento.

\section{Material e Métodos}

Área de estudo

A Bacia Hidrográfica do Riacho das Garças (BHRG) faz parte da rede de drenagem do Estado de Pernambuco, situando-se nas mesorregiões Sertão Pernambucano e São Francisco Pernambucano, tendo foz no Rio São Francisco (Figura 1). 
Figura 1 - Mapa de localização da Bacia Hidrográfica do Riacho das Garças (BHRG)

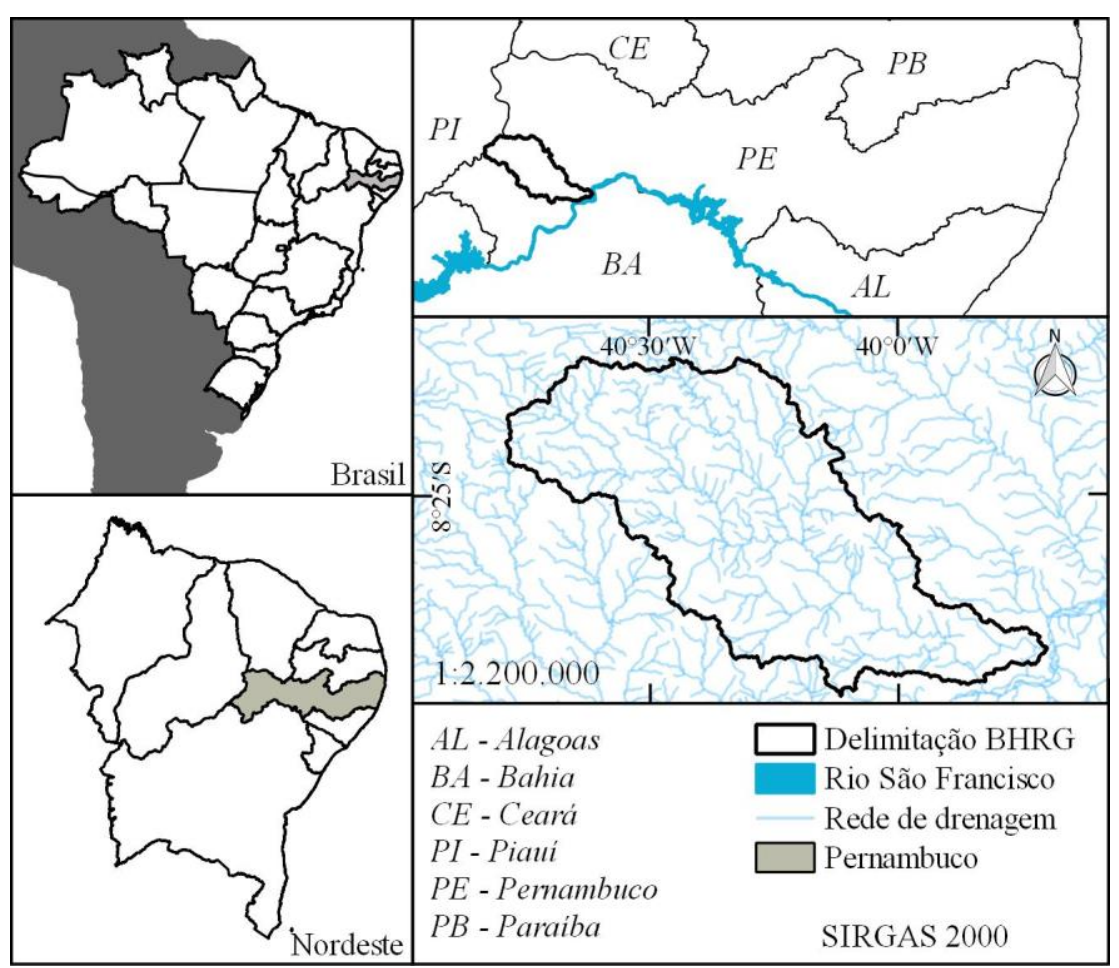

Fonte: Pesquisa direta.

Dentro do contexto semiárido nordestino, podem-se destacar as características de elevadas taxas de evapotranspiração e a consequente redução da umidade do solo e do volume de água armazenada, precipitações reduzidas e irregulares, que em conjunto promovem um balanço hídrico anual negativo (Correia et al., 2011). Além desses aspectos, a predominância de rochas cristalinas no Nordeste, que chega a ser mais que $80 \%$ de todo o solo dessa região, implica na baixa quantidade e qualidade das águas subterrâneas (Cirilo, 2008).

Além disso, em estudo sobre tendência de mudanças climáticas na pluviometria das bacias hidrográficas do Estado de Pernambuco, Silva et al. (2017), para BHRG, observaram uma tendência na diminuição da precipitação total anual, além do aumento no número de dias consecutivos secos e diminuição no número de dias consecutivos úmidos.

\section{Procedimentos realizados no QGIS}

O Software Quantum GIS (Geographic Information System), QGIS, versão 2.18.0, de acesso livre, foi utilizado como ferramenta de processamento.

Como Modelo Digital de Elevação - MDE foram utilizadas as imagens de elevação digital SRTM (Shuttle Radar Topography Mission) processadas e disponibilizadas pelo Laboratório de Geoprocessamento do Centro de Ecologia da Universidade Federal do Rio Grande do Sul (Weber et al., 2004), com resolução espacial de 90 metros. 0 processamento envolveu o preenchimento de falhas existentes nos dados originais por meio de interpolação, conversão para valores inteiros e 
ALVES, ATA; BARROS, VHO (2021)

caracterização morfométrica da Bacia Hidrográfica do Riacho das Garças, Pernambuco, Brasil

transformação ao Datum SAD 69 (South American Datum). No QGIS o arquivo raster foi reprojetado para SIRGAS 2000 e UTM 24 S e em seguida recortado para um polígono com menor área, mas que foi suficiente para abranger toda a bacia.

Para extração de informações do MDE foi utilizada a ferramenta TauDEM (Terrain Analysis Using Digital Elevation Models) - Utah State University, de acesso livre. Com essa ferramenta incorporada ao QGIS os passos seguidos foram: (i) obtenção do modelo digital de elevação hidrologicamente corrigido - pit remove; (ii) obtenção do raster de direção de fluxo preferencial na superfície - D8 flow Directions; (iii) construção da área de contribuição da bacia considerando o ponto exutório identificado - D8 contributing area; (iv) delimitação da rede de drenagem de fluxo canalizado - Stream definition by threshold; ( $v$ ) obtenção da rede de drenagem - Stream Reach and Watershed.

Em "Stream definition by threshold" foi utilizado o limiar 500, para o qual a rede de drenagem gerada coincidiu de forma aceitável com a hidrografia padrão da Base Cartográfica Contínua do Brasil, na escala 1:250.000 (IBGE, 2019).

Para obtenção das ordens dos cursos d'água foi utilizada a classificação "strmOrder". 0 arquivo em raster de delimitação da rede de drenagem foi transformado para o formato shapefile, mesclado suas feições e desse modo utilizado como camada máscara para recorte do SRTM para BHRG, necessário para análises do relevo. Para evitar a superestimação de valores no cálculo da área e do perímetro, foi realizada a suavização a partir do comando do GRASS "r.generalize.smooth" para as camadas vetoriais de delimitação das bacias.

Caracterização morfométrica de bacias hidrográficas

As variáveis morfométricas utilizadas para caracterizar a BHRG com as respectivas descrições/fórmulas são apresentadas na Tabela 1. Essas variáveis são relativas a características geométricas, de forma da bacia, de rede de drenagem e de relevo.

Tabela 1 - Características morfométrica selecionadas para análises

\begin{tabular}{|c|c|}
\hline Variável morfométrica & Descrição/Fórmula \\
\hline \multicolumn{2}{|r|}{ Características geométricas } \\
\hline $\begin{array}{l}\text { Área de drenagem }(\mathrm{A}) \text { - em } \\
\mathrm{km}^{2}\end{array}$ & Área de drenagem da bacia projetada em um plano horizontal \\
\hline Perímetro $(\mathrm{P})$ - em km & $\begin{array}{l}\text { Comprimento da linha ao longo do divisor de águas que delimita } \\
\text { área da bacia }\end{array}$ \\
\hline $\begin{array}{l}\text { Comprimento axial (L) - em } \\
\mathrm{km}\end{array}$ & $\begin{array}{l}\text { Comprimento da bacia medido do exutório até o ponto mais distan- } \\
\text { te }\end{array}$ \\
\hline \multicolumn{2}{|c|}{ Características de forma da bacia } \\
\hline $\begin{array}{l}\text { Coeficiente de compacida- } \\
\text { de }\left(\mathrm{K}_{\mathrm{c}}\right) \text { - adimensional }\end{array}$ & $\begin{array}{l}\text { Relação entre o perímetro da bacia e a } \\
\text { circunferência de um círculo de área igual } \\
\text { à da bacia (Villela \& Mattos, 1975; Horton, } \\
\text { 1932) }\end{array}$ \\
\hline $\begin{array}{l}\text { Fator de forma }\left(\mathrm{K}_{\mathrm{f}}\right) \text { - adi- } \\
\text { mensional }\end{array}$ & $\begin{array}{l}\text { Relação entre a largura média e o com- } \\
\text { primento axial da bacia (Horton, 1932) }\end{array}$ \\
\hline $\begin{array}{l}\text { Índice de circularidade }\left(\mathrm{I}_{c}\right) \\
\text { - adimensional }\end{array}$ & $\begin{array}{l}\text { Razão entre a área da bacia e a área de } \\
\text { um círculo com o mesmo perímetro da } \\
\text { bacia (Strahler, 1964) }\end{array}$ \\
\hline
\end{tabular}

Características da drenagem

Hierarquia hídrica - ordem $\quad$ Reflete o grau de ramificação de uma bacia (Strahler, 1957) 
ALVES, ATA; BARROS, VHO (2021)

caracterização morfométrica da Bacia Hidrográfica do Riacho das Garças, Pernambuco, Brasil

\begin{tabular}{|c|c|}
\hline $\begin{array}{l}\text { Comprimento total do flu- } \\
\text { xo de uma dada ordem - } \\
\text { em km }\end{array}$ & Comprimento do fluxo de uma ordem específica \\
\hline $\begin{array}{l}\text { Comprimento total dos } \\
\text { cursos d'água }\left(L_{t}\right)-\text { em km }\end{array}$ & Soma de todos os comprimentos dos cursos d'água \\
\hline $\begin{array}{l}\text { Comprimento do } \text { curso } \\
\text { d'água principal }\left(L_{P}\right)-\text { em } \\
\text { km }\end{array}$ & $\begin{array}{l}\text { Comprimento do curso principal que segue da nascente principal } \\
\text { até o exutório }\end{array}$ \\
\hline $\begin{array}{l}\text { Comprimento do talvegue } \\
\left(\mathrm{L}_{\text {tal }}\right) \text { - em km }\end{array}$ & $\begin{array}{l}\text { Comprimento em linha reta do canal principal seguindo da nascente } \\
\text { até o exutório }\end{array}$ \\
\hline $\begin{array}{l}\text { Sinuosidade do curso } \\
\text { d’água (Sin) - adimensional }\end{array}$ & $\begin{array}{l}\text { Relação entre o comprimento do rio prin- } \\
\text { cipal e o comprimento do talvegue (Villela } \\
\text { \& Mattos, 1975) }\end{array}$ \\
\hline $\begin{array}{l}\text { Densidade de drenagem } \\
\left(D_{d}\right)-\mathrm{em} \mathrm{km} \cdot \mathrm{km}^{-2}\end{array}$ & $\begin{array}{l}\text { Relação entre o comprimento total dos } \\
\text { cursos d'água de uma bacia e sua área } \\
\text { (Horton, 1932) }\end{array}$ \\
\hline $\begin{array}{l}\text { Densidade hidrográfica }\left(D_{h}\right) \\
\text { - em canais } \cdot \mathrm{km}^{-2}\end{array}$ & $\begin{array}{l}\text { Relação entre o número de cursos d'água } \\
\text { (n) e a área da bacia hidrográfica (Horton, } \\
\text { 1932) }\end{array}$ \\
\hline & Características do relevo \\
\hline $\begin{array}{l}\text { Altitudes e declividades } \\
\text { características }\end{array}$ & $\begin{array}{l}\text { Máxima e mínima, média, amplitude. Hipso } \\
\text { clividade. }\end{array}$ \\
\hline
\end{tabular}
Fonte: Pesquisa direta.

$\mathrm{Na}$ ordem dos cursos conforme Strahler (1957), canais sem tributários são considerados de primeira ordem; canais de segunda ordem resultam da confluência entre canais de primeira ordem; canais de terceira ordem resultam da confluência de canais de segunda ordem e assim sucessivamente; o encontro de canais de ordem diferentes resulta em um canal com a ordem daquele confluente com maior ordem.

\section{Resultados e discussão}

As características físicas de uma bacia hidrográfica atuam nos seus diversos processos hidrológicos, tais como velocidade de escoamento, infiltração e recarga do aquífero e evapotranspiração.

As características morfométricas da BHRG relativas à geometria, forma e rede de drenagem são apresentadas na Tabela 2. Em termos de geometria foi encontrada para BHRG uma área de $4097,205 \mathrm{~km}^{2}$, o perímetro de 412,242 km e o comprimento axial de 129,520 km. Pode-se dizer que a BHRG é de média dimensão.

Tabela 2 - Tabela resumo das características morfométricas obtidas para as bacias hidrográficas

\begin{tabular}{cc}
\hline Variável morfométrica & Valor \\
\hline $\mathrm{A}\left(\mathrm{km}^{2}\right)$ & 4097,205 \\
\hline $\mathrm{P}(\mathrm{km})$ & 412,242 \\
\hline $\mathrm{L}(\mathrm{km})$ & 129,520 \\
\hline $\mathrm{K}_{\mathrm{c}}$ & 1,803 \\
\hline $\mathrm{K}_{\mathrm{f}}$ & 0,244 \\
\hline $\mathrm{I}_{\mathrm{c}}$ & 0,303 \\
\hline Ordem & $5^{\circ}$ \\
\hline Comprimento & $1^{\circ}$ \\
\hline
\end{tabular}


ALVES, ATA; BARROS, VHO (2021)

caracterização morfométrica da Bacia Hidrográfica do Riacho das Garças, Pernambuco, Brasil

\begin{tabular}{ccc}
\hline \multirow{2}{*}{$\begin{array}{c}\text { total do fluxo de } \\
\text { uma dada ordem } \\
(\mathrm{km})\end{array}$} & $2^{\circ}$ & 371,406 \\
\cline { 2 - 3 } & $3^{\circ}$ & 230,609 \\
\cline { 2 - 3 } & $4^{\circ}$ & 46,325 \\
\hline $\mathrm{L}_{\mathrm{t}}(\mathrm{km})$ & $5^{\circ}$ & 113,139 \\
\hline $\mathrm{L}_{\mathrm{P}}(\mathrm{km})$ & 1494,577 \\
\hline $\mathrm{L}_{\mathrm{tal}}(\mathrm{km})$ & 192,288 \\
\hline Sin & 127,393 \\
\hline $\mathrm{D}_{\mathrm{d}}\left(\mathrm{km} \cdot \mathrm{km}^{-2}\right)$ & 1,509 \\
\hline $\mathrm{D}_{\mathrm{h}}\left(\right.$ canais $\left.\cdot \mathrm{km}^{-2}\right)$ & 0,365 \\
\hline
\end{tabular}

Fonte: Pesquisa direta.

$\mathrm{O}$ coeficiente de compacidade $\left(\mathrm{K}_{c}\right)$ expressa a relação entre a bacia hidrológica real e a bacia circular hipotética com a mesma área que a bacia hidrológica (Altaf et al., 2013) e varia com a forma da bacia independentemente de seu tamanho (Rocha et al., 2014) de modo que um coeficiente mínimo igual a unidade corresponde a uma bacia circular. A BHRG apresenta valor de $\mathrm{K}_{\mathrm{c}}$ relativamente distante da unidade $(1,803)$ indicando uma baixa tendência a ocorrência de enchentes pelo formato pouco circular que ela apresenta.

Outro fator que contribui para inferir a baixa tendência para enchentes é o fator de forma $\left(\mathrm{K}_{\mathrm{f}}\right)$, cujo valor para BHRG é de 0,244 , indicando, por ser menor que unidade, um formato mais alongado e uma baixa propensão a picos elevados de enchentes.

Além desses, o Índice de Circularidade $\left(I_{c}\right)$ também fornece um indicativo do formato maior ou menor para um círculo. Esse parâmetro tende para unidade à medida que a bacia hidrográfica se aproxima da forma circular, diminuindo à medida que a forma se torna alongada (Rocha et al., 2014). Para BHRG o valor encontrado para $I_{c}$, que foi de 0,303 , é distante da unidade e pode ser considerado baixo, indicando mais uma vez um formato alongado para BHRG, que favorece o escoamento superficial, e consequentemente, implica na baixa suscetibilidade a ocorrência de enchentes para BHRG

Com base nos valores encontrados para $\mathrm{K}_{c}, \mathrm{~K}_{f}$ e $\mathrm{I}_{c}$ em sub-bacias do município Xapuri, Amazônia, em que os valores variaram de 2,00 a 3,10 ( $\left.\mathrm{K}_{c}\right)$, 0,11 a 0,27 ( $\left.\mathrm{K}_{\mathrm{f}}\right)$ e 0,10 a 0,25 $\left(\mathrm{I}_{\mathrm{c}}\right)$, Abud et al., (2014) também aferiram a baixa propensão a ocorrência de inundações em condições normais de precipitação. Em estudo semelhante, mas na Bacia Hidrográfica do Rio Pontal, no extremo oeste do estado de Pernambuco, Lopes et al. (2018) encontraram os valores de 1,74, 0,41 e 0,32 para o $\mathrm{K}_{c}, \mathrm{~K}_{\mathrm{f}}$ e $\mathrm{I}_{\mathrm{c}}$, respectivamente, também indicando que essa bacia possui baixa suscetibilidade a enchentes. Entretanto, é importante destacar que não se pode avaliar a propensão à enchente em uma bacia exclusivamente por meio de sua forma (Lorenzon et al., 2015), já que outros fatores são atuantes, como características de cobertura do solo, grau de urbanização, dentre outros. De todo modo, esses resultados $\left(\mathrm{K}_{\mathrm{c}}, \mathrm{K}_{\mathrm{f}} \mathrm{e} \mathrm{I}_{\mathrm{c}}\right)$, juntamente com as características de precipitação do local, permitem apontar que a BHRG não apresenta grande potencialidade de ocorrência de enchentes em condições normais de precipitação.

A ordem dos cursos d'água conforme metodologia de Strahler (1957) indicou a $5^{\circ}$ ordem para BHRG. Em relação aos comprimentos totais do fluxo de uma dada ordem, os resultados, respectivamente para os cursos $1^{\circ}, 2^{\circ}, 3^{\circ}, 4^{\circ}$ e $5^{\circ}$ ordem, são: 733,099, 371,406, 230,609, 46,325, 113,139. o comprimento total dos cursos é $1494,6 \mathrm{~km}$. Quanto ao comprimento dos cursos de acordo 
ALVES, ATA; BARROS, VHO (2021)

caracterização morfométrica da Bacia Hidrográfica do Riacho das Garças, Pernambuco, Brasil

com as ordens é possivel verificar uma tendência de diminuição do comprimento com aumento das ordens, sendo o comprimento do fluxo máximo para primeira ordem.

O comprimento do rio principal $\left(L_{P}\right)$ e o comprimento do talvegue $\left(L_{\text {tal }}\right)$ da BHRG são, respectivamente, 192,288 e 127,393 km. O parâmetro sinuosidade do curso d'água (Sin) é de 1,509. O parâmetro Sin representa a geometria do canal e sinaliza se o canal é reto ou sinuoso (Rocha et al., 2014). O valor encontrado para BHRG indica a forma não retilínea de drenagem do canal principal.

Conforme Choudhari et al. (2018), geralmente a densidade de drenagem $\left(D_{d}\right)$ é dividida em quatro classes, variando de baixa $\left(D_{d}<2\right)$, moderada ( $D_{d}$ entre 2 e 4$)$, alta $\left(D_{d}\right.$ entre 4 e 6$)$ e muito alta ( $D_{d}$ acima de 6). Por essa classificação a $D_{d}$ da BHRG, $0,365 \mathrm{~km} \cdot \mathrm{km}^{-2}$, é considerada baixa. $A D_{d}$ é um importante fator na indicação do grau de desenvolvimento do sistema de drenagem (Rocha et al., 2014).

A densidade hidrográfica $\left(D_{h}\right)$ para BHRG foi de 0,135 canais $\cdot \mathrm{km}^{-2}$. Pode-se dizer que existe uma frequência aproximada de 0,14 canais para cada $\mathrm{km}^{2}$ de área, valor que pode ser considerado baixo e pode ter relação com o índice de precipitação da região associado aos elevados índices de evapotranspiração.

Com respeito ainda as características de drenagem, na Figura 2 é apresentado um mapa da rede de drenagem com a hierarquia hídrica para BHRG.

Figura 2 - Mapa da rede de drenagem com hieraquia hídrica conforme metodologia de Strahler (1957) para BHRG

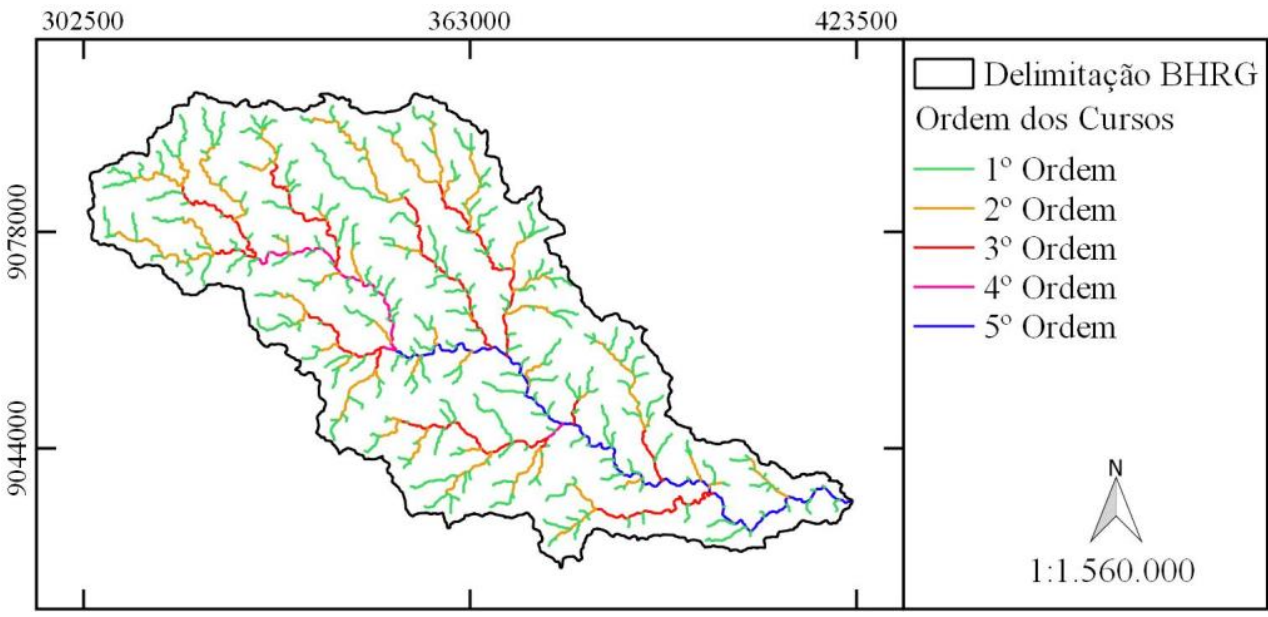

Fonte: Pesquisa direta.

Sobre as características do relevo, na Tabela 3 são apresentadas altitudes características para BHRG. Conforme é possível observar a altitude máxima é de $777 \mathrm{~m}$, mínima de $338 \mathrm{~m}$ com a amplitude de $439 \mathrm{~m}$. A média de altitudes ficou em torno de $476 \mathrm{~m}$.

Tabela 3 - Altitudes características obtidas para BHRG

\begin{tabular}{cccc}
\hline \multicolumn{4}{c}{ Altitude característica (m) } \\
\hline Máxima & Mínima & Amplitude & Média \\
\hline 777 & 338 & 439 & 475,858 \\
\hline
\end{tabular}


ALVES, ATA; BARROS, VHO (2021)

caracterização morfométrica da Bacia Hidrográfica do Riacho das Garças, Pernambuco, Brasil

Para melhora análise das altitudes da região, na Figura 3 é apresentado um mapa hipsométrico. As respectivas áreas e porcentagens relativas das classes são apresentadas na Tabela 4. Conforme é possível observar, a maior parte do relevo está compreendida na faixa de altitude de 400 a $500 \mathrm{~m}$, com porcentagem relativa de aproximadamente $58 \%$, sendo que a faixa acima de 700 $\mathrm{m}$ abrangeu menor área, menos de $0,5 \%$ em porcentagem. No mapa hipsométrico também é possível observar que as menores altitudes ficam na região da foz, no encontro da bacia com o Rio São Francisco, enquanto as maiores, na faixa $>700$, são verificadas apenas por pequenos pontos espaçados.

Figura 3 - Mapa hipsométrico para BHRG

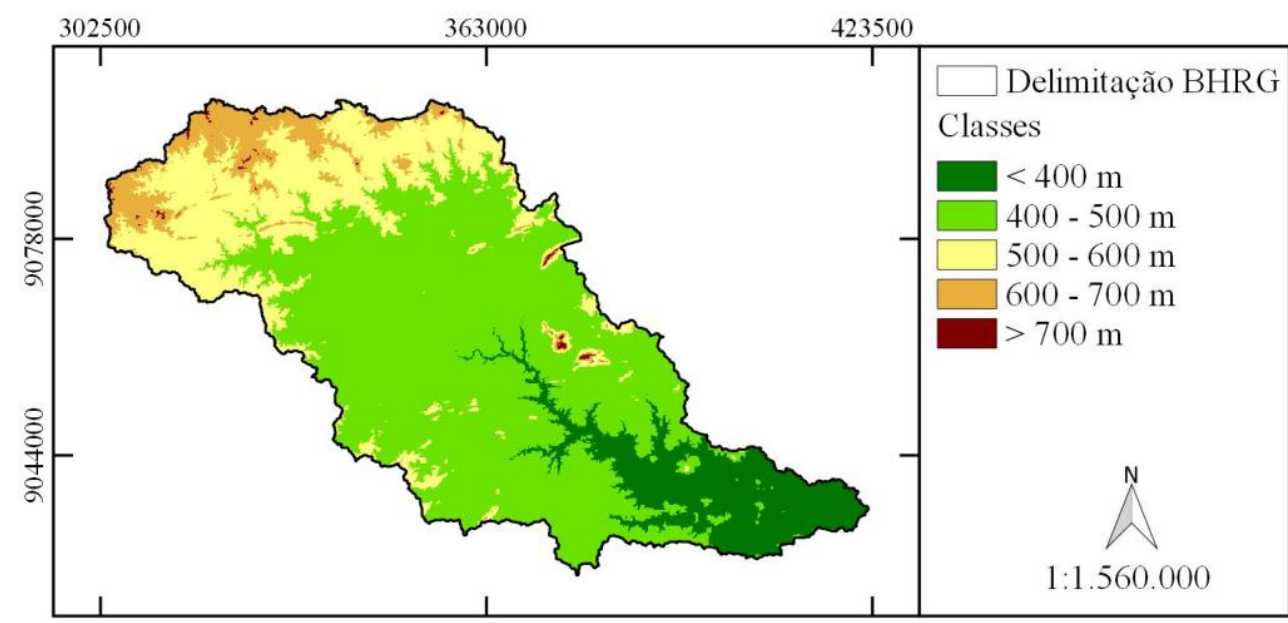

Fonte: Pesquisa direta.

Tabela 4 - Distribuição das classes de altimetria para BHRG

\begin{tabular}{ccc}
\hline Classes & \multicolumn{2}{c}{ Área } \\
\cline { 2 - 3 } & $\mathrm{km}^{2}$ & $\%$ \\
\hline Até $400 \mathrm{~m}$ & 510,284 & 12,458 \\
\hline Entre 400 e $500 \mathrm{~m}$ & 2386,134 & 58,256 \\
\hline Entre 500 e $600 \mathrm{~m}$ & 890,184 & 21,733 \\
\hline Entre 600 e $700 \mathrm{~m}$ & 295,918 & 7,225 \\
\hline Acima de $700 \mathrm{~m}$ & 13,393 & 0,327 \\
\hline Total & 4095,913 & 100 \\
\hline
\end{tabular}

Fonte: Pesquisa direta.

Sobre o aspecto declividade, um mapa conforme classes da EMBRAPA (2018) é apresentado na Figura 4. Na Tabela 5 são apresentadas as respectivas áreas e porcentagens relativas referentes a essas classes.

Figura 4 - Mapa de declividade do relevo para BHRG 
ALVES, ATA; BARROS, VHO (2021)

caracterização morfométrica da Bacia Hidrográfica do Riacho das Garças, Pernambuco, Brasil

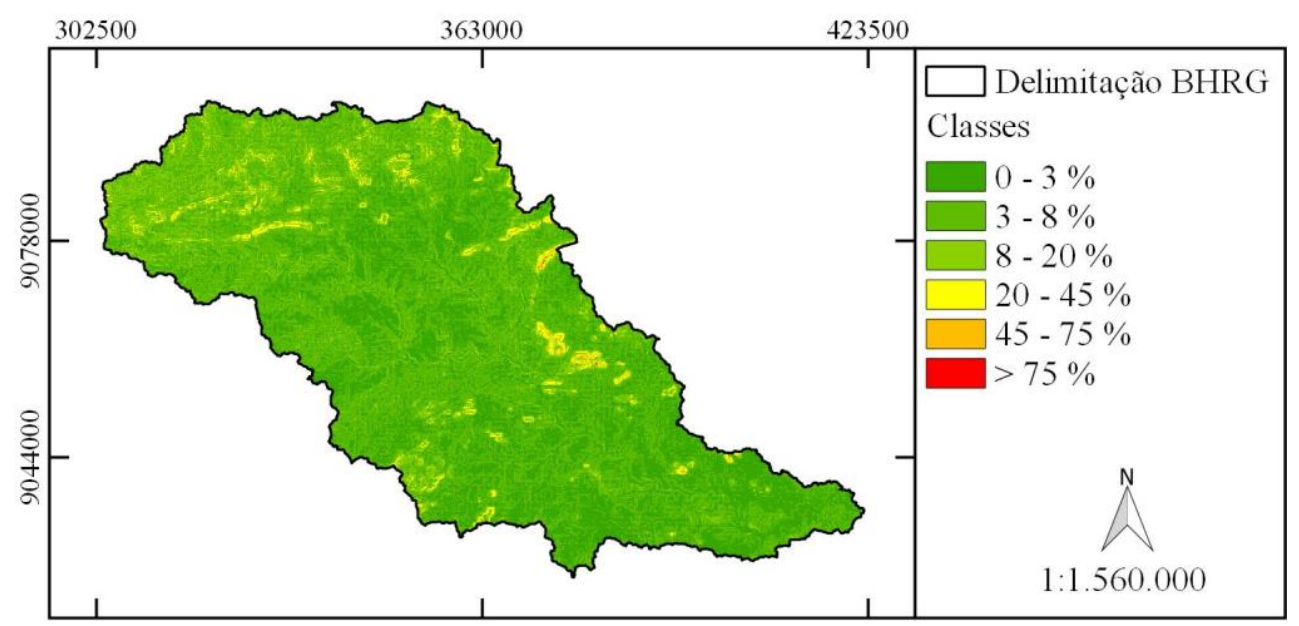

Fonte: Pesquisa direta.

Tabela 5 - Distribuição das classes de altimetria para BHRG

\begin{tabular}{cccc}
\hline Clas- & \multirow{2}{*}{ Relevo } & \multicolumn{2}{c}{ BHRG } \\
\cline { 3 - 4 } ses & & Área $\left(\mathrm{km}^{2}\right)$ & Área (\%) \\
\hline $0-3 \%$ & Plano & 1409,071 & 34,389 \\
\hline $3-8 \%$ & Suave ondulado & 2085,609 & 50,900 \\
\hline $8-20 \%$ & Ondulado & 509,606 & 12,437 \\
\hline $\begin{array}{c}20- \\
45 \%\end{array}$ & Forte ondulado & 86,864 & 2,120 \\
\hline $\begin{array}{c}45-75 \\
\%\end{array}$ & Montanhoso & 6,089 & 0,149 \\
\hline $\begin{array}{c}\text { Acima } \\
\text { de 75 \% }\end{array}$ & Escarpado & 0,251 & 0,006 \\
\hline Total & - & 4097,491 & 100,000 \\
\hline
\end{tabular}

Fonte: Pesquisa direta.

A declividade afeta a velocidade do escoamento superficial e a infiltração da água no solo. Se a declividade da região for acentuada, a água precipitada concentra-se mais rapidamente nos cursos dos rios aumentando as chances de ocorrer picos de enchentes (Lorenzon et al., 2015). Conforme é possível observar, o relevo da região é predominantemente plano $(34,389 \%)$ e suave ondulado (50,900\%), declividades mais acentuadas são pouco expressivas na região de estudo. Por essas características, o relevo possui uma topografia pouco movimentada com moderada velocidade de escoamento superficial, podendo ser aferido a baixa capacidade de erosão do solo além de um favorecimento da infiltração da água no solo.

\section{Conclusões}

Com base nos resultados obtidos, os valores encontrados para $\circ K_{c}, K_{f}$ e $I_{c}$ foram de 1,803, 0,244 e 0,303 , respectivamente, o que indica o formato alongado para BHRG e, consequentemente, favorece o escoamento superficial e a torna pouco propicia à ocorrência de enchentes em condições normais de precipitação. Em contrapartida, em relação à declividade da Bacia estudada, mais de $50 \%$ da área da bacia foi classificada com relevo suave ondulado, e quase $35 \%$ com relevo plano. Esse resultado indica que a inclinação da bacia não favorece o escoamento superficial. 
ALVES, ATA; BARROS, VHO (2021)

caracterização morfométrica da Bacia Hidrográfica do Riacho das Garças, Pernambuco, Brasil

Com base nos parâmetros relativos à drenagem, a $D_{d}$ da BHRG foi de $0,365 \mathrm{~km} \cdot \mathrm{km}^{-2}$, o que indica que a densidade de drenagem da bacia é baixa. Assim, foi possível concluir que a bacia hidrográfica em estudo se apresenta como pobremente drenadas.

Por fim, ficou claro com o estudo a facilidade de se estudar bacias hidrográficas por meio das ferramentas de geoprocessamento e sensoriamento remoto. Esse tipo de pesquisa é importante e pode auxiliar a identificação de áreas prioritárias em relação a diversos aspectos hidrológicos, como enchente e segurança hídrica, por exemplo, e auxiliar na tomada de decisão dos gestores e pesquisadores.

\section{Referências}

abdetA, G. C., TesemmA, A. B., TURA, A. L. \& ATLABACHEW, G. H. Morphometric analysis for prioritizing sub-watersheds and management planning and practices in Gidabo Basin, Southern Rift Valley of Ethiopia. Applied Water Science, 10:1-15, 2020.

ABUD, E. A., LANI, J. L., ARAÚJO, E. A., AMARAL, E. F., BARDALES, N. G. \& FERNANDES FILHO, E. I. F. Caracterização morfométrica das sub-bacias no município de Xapuri: subsídios à gestão territorial na Amazônia Ocidental. Revista Ambiente \& Água, 10:431-441, 2015.

ALTAF, F., MERAJ, G. \& ROMSHOO, S. A. Morphometric Analysis to Infer Hydrological Behaviour of Lidder Watershed, Western Himalaya, India. Geography Journal, 2013:1-14, 2013.

CARVALHO, A. A., LOPES, I., SILVA, O. F. Observed Trends in Precipitation over the Riacho do Navio Basin (Pernambuco). Anuário do Instituto de Geociências - Ufrj, 42:56-70, 2019.

CHOUDHARI, P.P., NIGAM, G.K., SINGH, S.K. \& THAKUR, S. Morphometric based prioritization of watershed for groundwater potential of Mula river basin, Maharashtra, India. Geology, Ecology, And Landscapes, 2:256-267, 2018.

CIRILO, J.A. Políticas públicas de recursos hídricos para o semiárido. Estudos Avançados, 22:61-82, 2008.

CORREIA, R. C., KIILL, L. H. P., MOURA, M. S. B., CUNHA, T. J. F., JESUS JÚNIOR, L. A. \& ARAUJO, J. L. P. A região semiárida brasileira. In: Voltolini, T. V. (ed.). Produção de caprinos e ovinos no Semiárido. Embrapa Semiárido, 2011. p.21-48.

EMBRAPA - Empresa Brasileira de Pesquisa Agropecuária. Sistema Brasileiro de Classificação de Solos. Brasília Embrapa, 2018. 356 p.

FIDELES, D. M., PIRES, G. T., CERIGNONE, F. J., TAVARES, C. V. G. \& RODRIGUES, V. A. Morphometric analysis of the watershed of Veloso and Café streams. Científica, 44:255-262, 2016. 
ALVES, ATA; BARROS, VHO (2021)

caracterização morfométrica da Bacia Hidrográfica do Riacho das Garças, Pernambuco, Brasil

HORTON, R.E. Drainage-basin characteristics. Transactions, American Geophysical Union, 13:350$361,1932$.

HORTON, R.E. Erosional development of streams and their drainage basins, hydrophysical approach to quantitative morphology. Bulletin of The Geological Society of America, 56:275-370, 1945.

IBGE. Instituto Brasileiro de Geografia e Estatística. Disponível em: <ftp://geoftp.ibge.gov.br/cartas_e_mapas/bases_cartograficas_continuas/bc250/versao2019/>. Acesso em: 05 jun. 2020.

LIRA, F. A., CARDOSO, A. de O. Estudo de tendência de vazões de rios das principais bacias hidrográficas brasileiras. Revista Brasileira de Ciências Ambientais, 21-37, 2018.

LOPES, I., RAMOS, C. M. C. \& LEAL, B. G. Caracterização morfométrica de bacia hidrográfica no semiárido de Pernambuco através de dados SRTM em softwares livre. Journal of Hyperspectral Remote Sensing, 8:31-40, 2018.

LORENZON, A. S., FRAGA, M. DE S., MOREIRA, A. R., ULIANA, E. M., SILVA, D. D. DA, RIBEIRO, C. A. A. S. \& BORGES, A. C. Influência das características morfométricas da bacia hidrográfica do rio Benevente nas enchentes no município de Alfredo Chaves-ES. Ambiente \& Água, 10:195-206, 2015.

MAHALA, A. (2019). The significance of morphometric analysis to understand the hydrological and morphological characteristics in two different morpho-climatic settings. Applied Water Science, 10:1-16, 2019.

OLIVEIRA, P. T. S., SOBRINHO, T. A., STEFFEN, J. L. \& RODRIGUES, D. B. B. Caracterização morfométrica de bacias hidrográficas através de dados SRTM. Revista Brasileira de Engenharia Agrícola e Ambiental, 14:819-825, 2010.

Rahaman, S. A., AJEeZ, S. A., ARUChamy, S. \& JeGankUmAR, R. Prioritization of Sub Watershed Based on Morphometric Characteristics Using Fuzzy Analytical Hierarchy Process and Geographical Information System - A Study of Kallar Watershed, Tamil Nadu. Aquatic Procedia, 4:1322-1330, 2015.

ROCHA, R. M., luCAS, A. A. T, AlmeidA, C. A. P., Menezes NetO, E. L. \& NetTo, A. O. A. Caracterização morfométrica da sub-bacia do rio Poxim-Açu, Sergipe, Brasil. Ambiente \& Água, 9:276-287, 2014.

SONI, S. Assessment of morphometric characteristics of Chakrar watershed in Madhya Pradesh India 
ALVES, ATA; BARROS, VHO (2021)

caracterização morfométrica da Bacia Hidrográfica do Riacho das Garças, Pernambuco, Brasil

using geospatial technique. Appl Water Sci, 7:2089-2102, 2017.

SILVA, R. O. B., MONTENEGRO, S. M. G. L. \& SOUZA, W. M. Tendências de mudanças climáticas na precipitação pluviométrica nas bacias hidrográficas do estado de Pernambuco. Engenharia Sanitária e Ambiental, 22:579-589, 2017.

SOUSA, F. R. C., PAULA, D. P. Caracterização morfométrica da bacia hidrográfica do rio Tapuio (Ceará-Brasil). Revista de Geociências do Nordeste, 2:555-564, 2016.

STRAHLER, A. N. Hypsometric (area-altitude) analysis of erosional topography. Geological Society of America Bulletin, 63:1117-1142, 1952.

STRAHLER, A. N. Quantitative analysis of watershed geomorphology. Transactions, American Geophysical Union, 38:913-920, 1957.

STRAHLER, A. N. (1964). Quantitative geomorphology of drainage basins and channel networks. In: CHOW, V.T. (ed.) Handbook of applied hydrology. McGraw Hill Book Company, 1964. p.439-476.

VILLELA, S.M. \& MATTOS, A. Hidrologia aplicada. São Paulo, Mcgraw-Hill do Brasil, 1975. 245 p.

WEBER, E., HASENACK, H. \& FERREIRA, C.J.S. Adaptação do modelo digital de elevação do SRTM para o sistema de referência oficial brasileiro e recorte por unidade da federação. Disponível em: <http://www.ecologia.ufrgs.br/labgeo>. Acesso em: 26 jun. 2020. 